\title{
D-Dimensional Log Gravity
}

\author{
Mohsen Alishahiha and Reza Fareghbal \\ School of physics, Institute for Research in Fundamental Sciences (IPM) \\ P.O. Box 19395-5531, Tehran, Iran \\ email: alishah@ipm.ir, fareghbal@theory.ipm.ac.ir
}

\begin{abstract}
We study Einstein gravity in dimensions $D \geq 4$ modified by curvature squared at critical point where the theory contains only massless gravitons. We show that at the critical point a new mode appears leading to a logarithmic gravity in the theory. The corresponding logarithmic solution may provide a gravity description of logarithmic CFT in higher dimensions. We note also that for special values of the parameters of the theory, the model admits solutions with non-relativistic isometries.
\end{abstract}




\section{Introduction}

Four dimensional Einstein gravity modified by curvature squared terms may provide a toy model to study quantum gravity in four dimensions. The corresponding action of the theory is given by [1, 2]

$$
I=\frac{1}{2 \kappa^{2}} \int \sqrt{-g} d^{4} x\left(R+\frac{6}{\ell^{2}}+\alpha R^{2}+\beta R^{\mu \nu} R_{\mu \nu}\right) .
$$

When the cosmological constant is zero, $\Lambda=0$, the theory is renormalizable and contains massless gravitons, massive spin 2 and a massive scalar fields [1,2]. Nevertheless the theory has ghosts due to negative energy excitations of the massive tensor. We note, however, that at the special values of the parameters of the theory either the massive tensor or the scalar would be absent. We note also that when $\beta=0$ the model is unitary but non-renormalizable, while when the curvature squared terms is given by the Weyl tensor squared the model is neither unitary nor renormalizable.

With non-zero cosmological constant the model exhibits new features. Although in this case the theory still contains massless gravitons, massive spin 2 and a massive

scalar fields, there is a possibility to tune the parameters such that only massless gravitons remain in the spectrum.

More precisely it has been shown [3] that for the special values of $\alpha$ and $\beta$ (critical point) given by

$$
\beta=-3 \alpha=-\frac{\ell^{2}}{2}
$$

the spin 2 field becomes massless and at the same time the massive scalar is removed from the spectrum. As a result we are left with four dimensional gravity with only massless gravitons.

In this letter we would like to further study the model at the critical point. In particular we study AdS wave solutions in the mode and we will show that at the critical point it admits logarithmic solutions. These solutions may provide gravity descriptions for logarithmic CFT's in higher dimensions.

The letter is organized as follows. In the next section we will consider AdS wave solutions in four dimensional theory at critical point. Generalization to higher dimensions is presented in section three. The last section is devoted to conclusions.

\section{AdS wave solution and Log gravity}

In this section we will study AdS wave solutions in the model given by the action (1.1). To procced we start with the equations of motion of the action (1.1)

$$
\mathcal{G}_{\mu \nu}+E_{\mu \nu}=0
$$


where [3]

$$
\begin{aligned}
\mathcal{G}_{\mu \nu} & =R_{\mu \nu}-\frac{1}{2} R g_{\mu \nu}-\frac{3}{\ell^{2}} g_{\mu \nu}, \\
E_{\mu \nu} & =2 \beta\left(R_{\mu \rho} R_{\nu}{ }^{\rho}-\frac{1}{4} R^{\rho \sigma} R_{\rho \sigma} g_{\mu \nu}\right)+2 \alpha R\left(R_{\mu \nu}-\frac{1}{4} R g_{\mu \nu}\right) \\
& +\beta\left(\square R_{\mu \nu}+\frac{1}{2} \square R g_{\mu \nu}-2 \nabla_{\rho} \nabla_{(\mu} R_{\nu}{ }^{\rho}\right)+2 \alpha\left(g_{\mu \nu} \square R-\nabla_{\mu} \nabla_{\nu} R\right) .
\end{aligned}
$$

Since $E_{\mu \nu}$ vanishes for any Einstein space in four dimensions, with a negative cosmological constant (as we choose here) the model admits an $\mathrm{AdS}_{4}$ vacuum solution whose radius is given by $\ell^{2}$. This solution has been studied in [3].

Having AdS vacuum solution, it is interesting to study AdS wave solutions in the mode 1 . To proceed we consider an anstaz for AdS wave solutions as follows

$$
g_{\mu \nu}=\bar{g}_{\mu \nu}+F k_{\mu} k_{\nu}
$$

where $k_{\mu}$ is a null vector field with respect to the metric $\bar{g}_{\mu \nu}$ with $\bar{g}_{\mu \nu}$ being the $\mathrm{AdS}_{4}$ metric parametrized as

$$
d s^{2}=\frac{\ell^{2}}{r^{2}}\left(-2 d x^{+} d x^{-}+d y^{2}+d r^{2}\right) .
$$

Note that $F$ is an arbitrary function which is independent of the integral parameter along $k_{\mu}$. In other words the ansatz may be given by

$$
d s^{2}=\frac{\ell^{2}}{r^{2}}\left(-F\left(x^{+}, y, r\right) d x^{+^{2}}-2 d x^{+} d x^{-}+d y^{2}+d r^{2}\right) .
$$

Plugging this ansatz into the equations of motion (2.1) one finds

$$
\begin{aligned}
{\left[\frac{\beta r^{3}}{\ell^{2}}\left(\frac{\partial^{4}}{\partial r^{4}}+2 \frac{\partial^{2}}{\partial y^{2}} \frac{\partial^{2}}{\partial r^{2}}+\frac{\partial^{4}}{\partial y^{4}}\right)\right.} & +\left(1-\frac{8(3 \alpha+\beta)}{\ell^{2}}\right) \\
& \left.\times\left(r \frac{\partial^{2}}{\partial r^{2}}-2 \frac{\partial}{\partial r}+r \frac{\partial^{2}}{\partial y^{2}}\right)\right] F\left(x^{+}, r, y\right)=0 .
\end{aligned}
$$

For simplicity we assume that $F$ is independent of $y$ coordinate. In this case the above equation reads

$$
\left[\frac{\beta r^{3}}{\ell^{2}} \frac{\partial^{4}}{\partial r^{4}}+\left(1-\frac{8(3 \alpha+\beta)}{\ell^{2}}\right)\left(r \frac{\partial^{2}}{\partial r^{2}}-2 \frac{\partial}{\partial r}\right)\right] F\left(x^{+}, r\right)=0 .
$$

It is clear that the most general solution of the above differential equation is in the form of $r^{x}$ with constant $x$ satisfying the following characteristic equation

$$
x(x-3)\left[\beta(x-1)(x-2)-8(3 \alpha+\beta)+\ell^{2}\right]=0 .
$$

\footnotetext{
${ }^{1}$ AdS wave solutions for TMG and NMG models have been studied in [4] where it was shown that at the critical value of the parameters the solution develops logarithmic behaviors. The same situation has also been observed in bi-gravity and Born-Infeld gravity [7,8].
} 
Therefore a generic solution of the equations of motion is 2

$$
F\left(x^{+}, r\right)=f_{4}\left(x^{+}\right)+f_{3}\left(x^{+}\right) r^{3}+f_{2}\left(x^{+}\right) r^{\Delta_{+}}+f_{1}\left(x^{+}\right) r^{\Delta_{-}} .
$$

where $\Delta_{ \pm}=\frac{3}{2} \pm \sqrt{\frac{9}{4}-\frac{\ell^{2}-24 \alpha-6 \beta}{\beta}}$, and $f_{i}$ 's are undetermined functions of $x^{+}$.

It is natural to look for a possibility of having multiplicities in the roots of the characteristic equation. Actually we observe that at the critical point where the parameters $\alpha$ and $\beta$ are given by the equation (1.2) the characteristic equation degenerates leading to new logarithmic solutions as follows

$$
F\left(x^{+}, r\right)=f_{4}\left(x^{+}\right)+f_{3}\left(x^{+}\right) r^{3}+\left[f_{2}\left(x^{+}\right)+f_{1}\left(x^{+}\right) r^{3}\right] \log (r) .
$$

In other words at the critical point the model admits a new vacuum solution which is not asymptotically locally $\mathrm{AdS}_{4}$. Therefore in order to accommodate the new solution one needs to change the asymptotic behavior of the metric. More precisely using the Fefferman-Graham coordinates for the metric

$$
d s^{2}=\frac{d r^{2}}{r^{2}}+\frac{1}{r^{2}} g_{i j}\left(x_{i}\right) d x^{i} d x^{j}
$$

the equations of motion give a possibility to have a wider class of boundary conditions for the metric as follows

$$
g_{i j}=b_{(0) i j} \log (r)+g_{(0) i j}+g_{(3) i j} r^{3}+b_{(3) i j} r^{3} \log (r)+\cdots .
$$

Typically when $b_{(0)}$ ij is non zero, where the solution is not an asymptotically locally $\mathrm{AdS}_{4}$, to maintain the variational principle well posed with the Dirichlet boundary condition one needs to modify the variational principle by imposing an additional boundary condition [10]. Indeed from the above expression for the asymptotic behavior of the metric one finds

$$
g_{(0) i j}=\lim _{r \rightarrow 0}\left(g_{i j}-r \log (r) \frac{\partial g_{i j}}{\partial r}\right), \quad b_{(0) i j}=\lim _{r \rightarrow 0} r \frac{\partial g_{i j}}{\partial r},
$$

showing that in order to fix the boundary conditions not only one needs the value of the boundary metric but also its radial derivative.

In the context of AdS/CFT correspondence [9] both $g_{(0) i j}$ and $b_{(0) i j}$ may be treated as two sources for two operators in the boundary 3-dimensional CFT. We note, however, that since in the presence of non-zero $b_{(0) i j}$ the geometry is not asymptotically locally $\mathrm{AdS}_{4}$, the parameter $b_{(0)}$ ij should be considered as a source for an irrelevant operator in the dual CFT (see for example [10]). Nevertheless for a sufficiently small $b_{(0) i j}$ one could still use the AdS/CFT correspondence to describe the dual theory which is expected to be a logarithmic CFT3.

\footnotetext{
${ }^{2} \mathrm{~A} y$ dependent solution can also be obtained as $F\left(x^{+}, y, r\right)=\left(c_{0}\left(x^{+}\right)+c_{1}\left(x^{+}\right) y\right) F\left(x^{+}, r\right)$.

${ }^{3}$ Logarithmic CFT's in the context of AdS/CFT correspondence have also been studied in [11]. We note, however, that in this paper the authors have fixed the background to be $A d S$ and the logarithmic behavior comes for the specific action of the fields in the bulk.
} 
As a result the logarithmic solution of the action (1.1) indicates that critical gravity gives a gravity description for three dimensional logarithmic CFT's. Actually the situation is similar to those in TMG and NMG models where it is believed that the dual theory is logarithmic CFT [12,13] at critical point. Following [10, 14] it would be interesting to find two point functions and the corresponding new anomaly parameter in this model using holographic renormalization method.

As a final remark we note that away from the critical point and for a specific values of $\alpha$ and $\beta$, the model admits another one parameter solution. Indeed when

$$
24 \alpha=2\left(2 n^{2}-n-4\right) \beta+\ell^{2},
$$

with $n$ being a free parameter, for a specific choice of the intergral constants one finds the following solution

$$
d s^{2}=\frac{\ell^{2}}{r^{2}}\left(-\frac{d t^{2}}{r^{2 n-2}}-2 d t d \xi+d y^{2}+d r^{2}\right) .
$$

We recognize this solution as a gravity solution whose dual theory is a non-relativistic field theory [15]. In particular for $n=2$ the isometry of the metric is Schrödinger group and the solution provide a gravity description for a non-relativistic CFT.

It is worth mentioning that in our model the non-relativistic holographic solution is obtained in a pure gravitational system, though the one studied in [15] has been obtained in a model which contains a gravity coupled to a massive gauge field. Of course we have not checked whether this vacuum is stable in the sense that small fluctuations above it have non-negative mass spectrum.

\section{Higher Dimensions}

In this section we would like to extend our previous discussions to higher dimensions. Recently D-dimensional extended gravities have been studied in [18] where the authors have considered the following gravitational action

$I=\frac{1}{\kappa} \int d^{D} x \sqrt{-g}\left[R-2 \Lambda_{0}+\alpha R^{2}+\beta R^{\mu \nu} R_{\mu \nu}+\gamma\left(R^{\mu \nu \rho \sigma} R_{\mu \nu \rho \sigma}-4 R^{\mu \nu} R_{\mu \nu}+R^{2}\right)\right]$.

The corresponding equations of motion are [19]

$$
\begin{aligned}
& R_{\mu \nu}-\frac{1}{2} g_{\mu \nu} R+\Lambda_{0} g_{\mu \nu}+2 \alpha R\left(R_{\mu \nu}-\frac{1}{4} g_{\mu \nu} R\right)+(2 \alpha+\beta)\left(g_{\mu \nu} \square-\nabla_{\mu} \nabla_{\nu}\right) R \\
& +2 \gamma\left[R R_{\mu \nu}-2 R_{\mu \sigma \nu \rho} R^{\sigma \rho}+R_{\mu \sigma \rho \tau} R_{\nu}^{\sigma \rho \tau}-2 R_{\mu \sigma} R_{\nu}{ }^{\sigma}-\frac{1}{4} g_{\mu \nu}\left(R_{\tau \lambda \sigma \rho}^{2}-4 R_{\sigma \rho}^{2}+R^{2}\right)\right] \\
& +\beta \square\left(R_{\mu \nu}-\frac{1}{2} g_{\mu \nu} R\right)+2 \beta\left(R_{\mu \sigma \nu \rho}-\frac{1}{4} g_{\mu \nu} R_{\sigma \rho}\right) R^{\sigma \rho}=0 .
\end{aligned}
$$

\footnotetext{
${ }^{4}$ Lifshitz black holes in Einstein gravity with curvature squared terms have also been studied in 16,17 .
} 
For generic values of the parameters $\Lambda, \alpha, \beta$ and $\gamma$ the model has two distinct vacua such that $R_{\mu \nu}=\frac{2 \Lambda}{D-2} g_{\mu \nu}$, where $\Lambda$ is a root of the following equation [18]

$$
\Lambda_{0}-\Lambda=2 \Lambda^{2}\left[(D \alpha+\beta) \frac{D-4}{(D-2)^{2}}+\frac{(D-3)(D-4)}{(D-1)(D-2)} \gamma\right] .
$$

It is always possible to tune the parameters such that at least one of the vacua to be an $\mathrm{AdS}_{D}$ geometry. In this case the radius of the AdS geometry is given in terms of $\Lambda$ as follow 5

$$
\ell^{2}=-\frac{(D-1)(D-2)}{2 \Lambda}
$$

It was shown in [18] that for appropriate choice of the parameters there exists a critical point where

$$
\beta=-\frac{4(D-1)}{D} \alpha, \quad \frac{(D-1)(D-2)}{4(-\Lambda)}=(D-1)(D \alpha+\beta)+(D-3)(D-4) \gamma
$$

at which the model has only massless tensor gravitons.

Following our discussions in the previous section it is natural to look for AdS wave solutions in the model and in particular to see if the model supports a logarithmic solution at the critical point. To proceed we consider an ansatz as follows

$$
d s^{2}=\frac{\ell^{2}}{r^{2}}\left(-F\left(x^{+}, r, x_{i}\right) d u^{2}-2 d x^{+} d x^{-}+d r^{2}+\left(d x_{i}\right)^{2}\right) .
$$

Plugging this ansatz into the equations of motion one finds

$$
\begin{aligned}
& \left\{\frac{\beta r^{3}}{D-2}\left(\frac{\partial^{2}}{\partial r^{2}}+\frac{\partial^{2}}{\partial x_{i}^{2}}\right)^{2}-\frac{2(D-4)}{D-2} \beta r^{2}\left(\frac{\partial^{2}}{\partial r^{2}}+\frac{\partial^{2}}{\partial x_{i}^{2}}\right) \frac{\partial}{\partial r}+\beta r(D-4) \frac{\partial^{2}}{\partial r^{2}}(3.7)\right. \\
& \quad+\frac{r}{D-2}\left[\ell^{2}-2 D(D-1) \alpha-4(D-2) \beta-2(D-3)(D-4) \gamma\right]\left(\frac{\partial^{2}}{\partial r^{2}}+\frac{\partial^{2}}{\partial x_{i}^{2}}\right) \\
& \left.-\left[\ell^{2}-2 D(D-1) \alpha-(3 D-4) \beta-2(D-4)(D-3) \gamma\right] \frac{\partial}{\partial r}\right\} F\left(x^{+}, r, x_{i}\right)=0 .
\end{aligned}
$$

The equation may be simplified with the assumption that the function $F$ is independent of transverse directions $x_{i}$ 's. In this case a generic solution of the resultant equation will be of the form $r^{x}$ for constant $x$. From the above equation the characteristic equation reads

$$
\frac{\beta}{D-2} x(x-D+1)\left(x^{2}-(D-1) x+\frac{A}{\beta}\right)=0
$$

\footnotetext{
${ }^{5}$ When the right hand side of the equation (3.3) vanishes with the assumption of negative cosmological constant, $\Lambda=\Lambda_{0}<0$, the model admits a unique AdS solution. In this case when $D \neq 4$ the parameters of the model have to obey the constraint $D \alpha+\beta+\frac{(D-2)(D-3)}{D-1} \gamma=0$.
} 
where $A=l^{2}-2(D \alpha+\beta)(D-1)-2(D-3)(D-4) \gamma$.

Therefore the most general solution of the equations of motion is

$$
F\left(x^{+}, r\right)=f_{4}\left(x^{+}\right)+f_{3}\left(x^{+}\right) r^{D-1}+f_{2}\left(x^{+}\right) r^{\Delta_{+}}+f_{1}\left(x^{+}\right) r^{\Delta_{-}},
$$

where $\Delta_{ \pm}=\frac{D-1}{2} \pm \sqrt{\left(\frac{D-1}{2}\right)^{2}-\frac{A}{\beta}}$, and $f_{i}$ 's are undetermined functions of $x^{+}$.

We note that at the critical point (3.5) where $A=0$ the characteristic equation degenerates leading to new logarithmic solutions as follows

$$
F\left(x^{+}, r\right)=f_{4}\left(x^{+}\right)+f_{3}\left(x^{+}\right) r^{D-1}+\left[f_{2}\left(x^{+}\right)+f_{1}\left(x^{+}\right) r^{D-1}\right] \log (r) .
$$

As a results, following our discussions in the previous section, the gravitational model based on the action (3.1) at the critical point may provide a gravity description for $D-1$ dimensional logarithmic CFT's.

The model also admits non-relativistic solutions when the parameters of the model obey the constraint $A+2(n-1)(2 n-D-1) \beta=0$ for $n \neq 1$. In this case for special choice of the integral constnats one finds

$$
d s^{2}=\frac{\ell^{2}}{r^{2}}\left(-\frac{d t^{2}}{r^{2 n-2}}-2 d t d \xi+d r^{2}+\left(d x_{i}\right)^{2}\right) .
$$

\section{Conclusions}

In this letter we have studied AdS wave solutions in $D$-dimensional Einstein gravity with curvature squared modification. At the critical point where the theory contains only massless gravitons the model admits logarithmic solutions.

We have also shown that for special values of the parameters of the model, one could have non-relativistic solutions. In particular the model admits solutions with Schrödinger isometry. Therefore these models could provide gravity descriptions for non-relativistic and logarithmic CFT's.

It is important to note that the existence of these solutions are due to the curvature squared terms in the action. Actually restricting to four dimensions we note that any Einstein solutions are the solutions of the model with curvature squared action. In fact black hole solutions of the Eisntein kind have altready been discussed in [3] where the authors have shown that the corresponding black holes have zero mass and entropy!

It would be interesting to find other solutions in the model which are not Einstein solutions. In particular one may seek for non-Einstein black hole solutions in the model. These black holes could be of the logarithmic solution as well.

Note added: After we submitted our paper to arXiv, two other papers, 20] and [21], appeared in arXiv where the similar logarithmic solutions have been discussed. It was also conjectured that the corresponding dual field theory could be a logarithmic CFT. 


\section{Acknowledgments}

We would like to thank Ali Naseh for discussions. This work is supported by Iran National Science Foundation (INSF). We would also like to thank referee for his/her useful comments.

\section{References}

[1] K. S. Stelle, "Renormalization Of Higher Derivative Quantum Gravity," Phys. Rev. D 16, 953 (1977).

[2] K. S. Stelle, "Classical Gravity With Higher Derivatives," Gen. Rel. Grav. 9, 353 (1978).

[3] H. Lu and C. N. Pope, "Critical Gravity in Four Dimensions," arXiv:1101.1971 [hep-th].

[4] E. Ayon-Beato and M. Hassaine, "pp waves of conformal gravity with selfinteracting source," Annals Phys. 317, 175 (2005) arXiv:hep-th/0409150.

[5] E. Ayon-Beato and M. Hassaine, "Exploring AdS waves via nonminimal coupling," Phys. Rev. D 73, 104001 (2006) arXiv:hep-th/0512074.

[6] E. Ayon-Beato, G. Giribet and M. Hassaine, "Bending AdS Waves with New Massive Gravity," JHEP 0905, 029 (2009) [arXiv:0904.0668 [hep-th]].

[7] H. R. Afshar, M. Alishahiha and A. Naseh, "On three dimensional bigravity," Phys. Rev. D 81, 044029 (2010) [arXiv:0910.4350 [hep-th]].

[8] M. Alishahiha, A. Naseh and H. Soltanpanahi, "On Born-Infeld Gravity in Three Dimensions," Phys. Rev. D 82, 024042 (2010) arXiv:1006.1757 [hepth]].

[9] J. M. Maldacena, "The large N limit of superconformal field theories and supergravity," Adv. Theor. Math. Phys. 2, 231 (1998) [Int. J. Theor. Phys. 38, 1113 (1999)] arXiv:hep-th/9711200].

[10] K. Skenderis, M. Taylor and B. C. van Rees, "Topologically Massive Gravity and the AdS/CFT Correspondence," JHEP 0909, 045 (2009) arXiv:0906.4926 [hep-th]].

[11] A. M. Ghezelbash, M. Khorrami and A. Aghamohammadi, "Logarithmic conformal field theories and AdS correspondence," hep-th/9807034.

[12] D. Grumiller and N. Johansson, "Instability in cosmological topologically massive gravity at the chiral point," JHEP 0807, 134 (2008) arXiv:0805.2610 [hepth]]. 
[13] D. Grumiller and O. Hohm, "AdS $3 / \mathrm{LCFT}_{2}$ - Correlators in New Massive Gravity," Phys. Lett. B 686, 264 (2010) arXiv:0911.4274 [hep-th]].

[14] M. Alishahiha and A. Naseh, "Holographic Renormalization Of New Massive Gravity," Phys. Rev. D 82, 104043 (2010) [arXiv:1005.1544 [hep-th]].

[15] D. T. Son, "Toward an AdS/cold atoms correspondence: a geometric realization of the Schroedinger symmetry," Phys. Rev. D 78, 046003 (2008) arXiv:0804.3972 [hep-th]].

[16] R. G. Cai, Y. Liu and Y. W. Sun, "A Lifshitz Black Hole in Four Dimensional $R^{2}$ Gravity," JHEP 0910, 080 (2009) arXiv:0909.2807 [hep-th]].

[17] E. Ayon-Beato, A. Garbarz, G. Giribet and M. Hassaine, "Analytic Lifshitz black holes in higher dimensions," JHEP 1004, 030 (2010) arXiv:1001.2361 [hep-th]].

[18] S. Deser, H. Liu, H. Lu, C. N. Pope, T. C. Sisman and B. Tekin, "Critical Points of D-Dimensional Extended Gravities," arXiv:1101.4009 [hep-th].

[19] I. Gullu and B. Tekin, "Massive Higher Derivative Gravity in D-dimensional Anti-de Sitter Spacetimes," Phys. Rev. D 80, 064033 (2009) arXiv:0906.0102 [hep-th]].

[20] I. Gullu, M. Gurses, T. C. Sisman and B. Tekin, "AdS Waves as Exact Solutions to Quadratic Gravity," arXiv:1102.1921 [hep-th].

[21] E. A. Bergshoeff, O. Hohm, J. Rosseel and P. K. Townsend, "Modes of Log Gravity," arXiv:1102.4091 [hep-th]. 